\title{
Nordiques
}

36 | 2018

Réformer l'éducation en Europe du Nord

\section{Nicolaj Frederik Severin Grundtvig, L'école pour la vie}

Vrin (Philosophie de l'éducation), 2018

Michel Forget

\section{CpenEdition}

\section{Journals}

Édition électronique

URL : http://journals.openedition.org/nordiques/541

DOI : 10.4000/nordiques.541

ISSN : 2777-8479

Éditeur :

Association Norden, Bibliothèque de Caen la mer

Édition imprimée

Date de publication : 1 novembre 2018

Pagination : 131-135

ISBN : 9791095914020

ISSN : 1761-7677

\section{Référence électronique}

Michel Forget, « Nicolaj Frederik Severin Grundtvig, L'école pour la vie », Nordiques [En ligne], 36 | 2018, mis en ligne le 31 octobre 2020, consulté le 13 mars 2021. URL : http://journals.openedition.org/ nordiques/541 ; DOI : https://doi.org/10.4000/nordiques.541

Ce document a été généré automatiquement le 13 mars 2021.

Nordiques 


\section{Nicolaj Frederik Severin Grundtvig, L'école pour la vie}

Vrin (Philosophie de l'éducation), 2018

Michel Forget

\section{RÉFÉRENCE}

Nicolaj Frederik Severin Grundtvig, L'école pour la vie, Vrin (Philosophie de l'éducation), 2018, Textes présentés par Jean-François Dupeyron, Christophe Miqueu et France Roy, traduction et notes de Marc Auchet, avant-propos d'Ove Korsgaard

1 Au Danemark, la période 18001850, en dépit de la situation désastreuse du pays du point de vue économique et politique (guerres napoléoniennes, incendies, défaites, banqueroute de l'État...) a été nommée l'Âge d'or en raison de l'extraordinaire floraison d'artistes ou d'auteurs qui se sont illustrés alors dans tous les domaines (science, littérature, peinture, musique théâtre...). Dans celui de la pensée, trois noms dominent tous les autres : Søren Kierkegaard (1813-1855), Hans Christian Andersen (1805-1875) et Nicolaj Frederik Severin Grundtvig (1783-1872). Alors que les deux premiers ont franchi les frontières de leur pays natal, le troisième n'a pas eu cette chance. Pasteur, théologien, historien, philologue, poète, auteur de cantiques luthériens ou théoricien de l'éducation, son œuvre protéiforme a pourtant marqué durablement la culture de son pays jusqu'à aujourd'hui. Cependant Grundtvig demeure pratiquement inconnu en dehors du Danemark et des pays scandinaves, en France en tout cas.

C'est pourquoi l'anthologie de textes de Grundtvig, coordonnée par Marc Auchet et que viennent d'éditer France Roy, Jean-François Dupeyron et Christophe Miqueu constitue, en soi, un véritable événement qui vient combler partiellement une absence regrettable dans le champ éditorial francophone.

Les textes retenus ont pour dénominateur commun les idées de Grundtvig sur l'école et sur l'éducation. Celles-ci sont inséparables de sa réflexion théologique et s'en déduisent comme leur aboutissement logique. En effet, l'une des grandes idées autour desquelles 
va se construire son œuvre est celle de la supériorité du verbe sur la chose écrite et, par conséquent, celle de la prééminence de la tradition orale, de la parole vivante qui se transmet d'homme à homme sur l'enseignement livresque et la sèche érudition. Cette supériorité de la transmission par la parole et l'échange implique que soit donnée priorité à l'enseignement oral et vivant et suppose ainsi une nouvelle manière d'enseigner non seulement aux enfants, mais également aux adultes. C'est en suivant jusqu'à ses conséquences extrêmes cet axe de réflexion que Grundtvig en viendra à développer ses propositions pour la création des hautes écoles populaires auxquelles son nom reste attaché.

4 L'ouvrage s'ouvre par un avant-propos d'Ove Korsgaard ${ }^{1}$ qui situe la vie et l'œuvre de Grundtvig dans leur contexte, présente brièvement les principaux thèmes grundtvigiens dont la connaissance facilitera la lecture des développements ultérieurs et signale l'influence durable de cette pensée en Scandinavie et dans le reste du monde.

De son côté, Marc Auchet attire l'attention sur la difficulté de la traduction de la prose de Grundtvig dont le style poétique est quelquefois alambiqué, la syntaxe complexe, les allusions multiples à des épisodes mal connus chez nous de la mythologie nordique, rendent la lecture de ses textes parfois ardue et expliquent, au moins partiellement, qu'ils aient eu du mal à trouver leur chemin en dehors de leur sphère d'origine.

6 L'ouvrage proprement dit consiste en une anthologie réunissant six textes de Grundtvig, brièvement présentés chacun par l'un ou l'autre des éditeurs du volume. Bien que la pensée éducative de Grundtvig ne concerne pas principalement l'école élémentaire, celui-ci ne se prive pas, au fil des pages, de dénoncer le caractère mortifère de l'école traditionnelle, avec sa prééminence donnée au latin, à la grammaire et aux mathématiques au détriment de connaissances directement utiles aux enfants. Il a, pour dénoncer cette "école pour la mort» (p.4), avec cent ans d'avance, des accents que n'aurait pas désavoués un Célestin Freinet, chez nous :

Si [...] nous voulons inculquer aux enfants l'ordre, le calme, la réflexion et la sagesse de la vieillesse, nous ne faisons que leur inculquer la mort de l'âme et du corps qui provient de la faiblesse de la vieillesse. Chez un grand nombre d'entre eux, nous ne faisons qu'anéantir toute force vitale, si bien qu'ils s'estompent comme des ombres, alors qu'ils ne sont encore que des adolescents, et le travail que nous faisons en eux tous détruit la nature humaine en s'opposant à ses lois. (p. 41)

7 Le texte La patrie et la langue maternelle, présenté par France Roy, fait partie d'un ensemble plus vaste que Grundtvig avait intitulé Le trèfle à quatre feuille danois. L'écrivain y exprime son amour d'un Danemark symbolisé poétiquement sous la forme d'un trèfle dont chaque feuille représente le roi, le peuple, la patrie et la langue maternelle. "J'ai encore dans la bouche le goût empoisonné du latin ", écrit-il, c'est pourquoi il mise, au contraire, sur la langue maternelle, le danois, seule capable de faire naître une culture partagée, conforme à l'esprit du peuple et à ses besoins.

Dans le texte intitulé L'éducation pour l'État (1834), Grundtvig développe, entre autres, cette idée révolutionnaire pour l'époque selon laquelle l'éducation doit s'étendre à la vie tout entière et concerner tous les cercles de la société « depuis les gens du commun jusqu'aux plus érudits » (p.102). Il est intéressant de voir cette idée, pour nous relativement récente, d'éducation permanente, apparaitre dès 1834 sous la plume de Grundtvig. Ce n'est d'ailleurs pas un hasard si la Communauté européenne a choisi de donner le nom de Grundtvig à son programme éducatif relatif à l'éducation des adultes 
tout au long de la vie (Life long learning), reconnaissance tardive mais bienvenue de la vision prophétique du penseur danois.

D'un texte à l'autre, Grundtvig développe, sous des formes variées, ce qu'il entend par ces «hautes écoles populaires» (folkehøjskoler) dont il souhaite la création et la généralisation dans tout le pays. L'un des plus explicites est celui intitulé Idée d'une haute école royale danoise. Sachant le roi Christian VIII favorable à ses idées pédagogiques, Grundtvig profite de son accession au trône pour lui adresser une longue missive dans laquelle il développe et détaille ce qu'il entend par «haute école populaire » et son désir de voir l'ancienne académie de Sorø être transformée en ce sens. On sait que la mort prématurée du roi ne permit pas l'aboutissement de ce projet. Mais les idées de Grundtvig à ce sujet connurent une diffusion rapide dans tout le pays comme en Suède ou en Finlande, et de nombreuses écoles virent bientôt le jour dont on peut dire que Grundtvig en fut le père spirituel et l'instigateur, fût-ce de façon indirecte.

10 Dans ce long texte, Grundtvig ironise une fois de plus sur "les érudits qui croient encore que la grammaire latine est la pierre philosophale et que la composition latine permet d'être inscrit dans le livre des êtres pensants» (p. 132). "J'ai la conviction absolue, et, j'ose dire, bien fondée, que toute cette science pour collégiens byzantine et tout ce système chinois d'examens sont d'une part tellement vides et d'autre part tellement peu naturels et extravagants que ce sera un triomphe pour l'esprit humain, un gain et une joie pour tous les âges si tout cela tombe dans l'oubli chez nous " (p. 132-133). À l'inverse, une haute école populaire devrait être, selon lui, «un établissement d'enseignement par lequel le peuple s'éveillerait et prendrait petit à petit conscience de lui-même, et où les dirigeants apprendraient autant de la jeunesse que celle-ci apprendrait d'eux, un échange vivant et un enseignement réciproque grâce auquel un pont serait jeté au-dessus de l'abîme béant que la hiérarchie, l'aristocratie, le latin et l'honnête ambition ont creusé entre, d'une part, presque tout le peuple, et, d'autre part, ses dirigeants et enseignants... » (p. 138). Il s'agirait évidemment d'une école sans examens, car «ce qu'on apprend à cause d'un examen ou d'un gagne-pain, on se hâte évidemment de l'oublier une fois l'examen passé et le gagne-pain obtenu » (p. 147). Parmi les matières d'enseignement l'histoire et la poésie, la connaissance du patrimoine auraient naturellement ici une place privilégiée.

11 Cette promotion de l'enseignement oral avait aussi une dimension politique. Grundtvig s'est toujours montré méfiant à l'égard de l'émergence des tendances démocratiques et il est resté attaché aux valeurs d'une monarchie absolue éclairée, mais il n'était pas insensible aux aspirations profondes qui travaillaient le Danemark et toute l'Europe à cette époque. C'est pourquoi il voyait aussi dans l'enseignement oral qu'il préconisait un moyen offert à chacun de maîtriser progressivement sa parole et son expression y compris dans les conseils du peuple prévus dans le cadre des assemblées provinciales consultatives créées par Frederik VI et dans lesquels commençaient à s'exprimer au grand jour les aspirations démocratiques et le désir de plus en plus pressant d'une Constitution.

12 Le livre s'achève par deux «contributions annexes » importantes et qui constituent d'excellentes synthèses des idées de Grundtvig sur l'éducation et des questions soulevées par son œuvre. Dans la première, N. F. S Grundtvig. Un éducateur pour la vie, Jean-François Dupeyron s'attache d'abord à une comparaison des écoles danoise et française actuelles. Il y décrit l'opposition entre la rigidité, l'élitisme et le primat de la 
notation qui sont ceux de l'école en France et l'ambiance générale de l'école danoise dans laquelle le souci principal est «d'aider l'enfant à se familiariser avec la vie en collectivité et à s'épanouir dans cet ensemble culturel, politique et social que constitue la démocratie danoise " (p. 208). L'auteur présente ensuite deux structures originales du système éducatif danois actuel : l'efterskole et la folkehøjskole. La première dont le nom signifie "l'école d'après" désigne un établissement facultatif que peuvent fréquenter les enfants qui ont achevé leur scolarité primaire avant d'entrer dans le secondaire ou les études professionnelles. La vie en internat y favorise l'apprentissage de la vie démocratique plutôt que la compétition scolaire permanente. Une ou deux années passées dans un établissement de ce type permettent, en outre, aux élèves une découverte des études ou des apprentissages vers lesquels ils pourraient ensuite s'orienter. La folkehøjskole (ou haute école populaire), quant à elle, est un dispositif directement inspiré par les idées de Grundtvig. Sans notation, sans délivrance de diplômes, sans scolastique (un mot familier à Célestin Freinet), les folkehøjskoler se veulent des sortes "d'universités pour le peuple ». Ouvertes à tous, gratuitement, elles constituent une possibilité offerte, à chacun, tout au long de sa vie, d'une formation permanente d'adultes dans les domaines de son choix. Ce dispositif original dont le modèle est directement inspiré des idées de Grundtvig s'est répandu également dans d'autres pays scandinaves même si les acteurs actuels du système ignorent ou ont oublié son origine ${ }^{2}$.

La seconde " contribution annexe » due à Christophe Miqueu, intitulée L'école du peuple, s'attache, par un examen attentif des textes, à comparer l'école du peuple selon Grundtvig avec les fondements de l'école républicaine en France. Cette analyse fait apparaître les convergences, mais surtout les divergences de ces deux approches philosophiques de l'éducation: primat du rationnel et recherche de l'émancipation intellectuelle d'un côté, enracinement dans la culture danoise de l'autre; priorité à l'égalité d'éducation pour l'école républicaine, privilège accordé à la liberté que donne l'éducation entendue d'abord comme partage d'une culture populaire commune enracinée dans le patrimoine culturel nordique, pour l'école danoise. Même sur la question de l'exclusion de la religion de la sphère scolaire, l'apparente convergence des points de vue est trompeuse et répond à des préoccupations différentes. Analysant le dernier texte de Grundtvig retenu dans cette anthologie intitulé La foi est-elle vraiment une matière scolaire?, Christophe Miqueu souligne avec pertinence les différences irréductibles entre la laïcité à la française et l'exclusion du religieux de la sphère scolaire par Grundtvig, les deux écoles obéissant ici à des préoccupations diamétralement opposées. Pour l'école républicaine il s'agissait en effet avant tout de protéger les enfants de l'emprise d'un catholicisme jugé envahissant, alors que pour Grundtvig, le refus de l'enseignement religieux à l'école était motivé, au contraire, par sa volonté de défendre un enseignement chrétien authentique menacé, selon lui, par des maîtres sans foi véritable ou trop sensibles aux séductions du rationalisme hérité des Lumières et dont l'influence sur les élèves risquait de les conduire vers l'incrédulité.

14 Il est impossible de passer en revue, en quelques pages, l'ensemble des problèmes abordés par ces textes de Grundtvig que les auteurs ont eu le mérite de tirer de l'oubli et de porter enfin à la connaissance du public francophone. Certes, il ne s'agit ici que de textes où s'expriment les idées philosophiques de Grundtvig sur l'éducation, mais les différentes présentations qui en sont données, les notes et les deux «contributions annexes » sur lesquelles se referme le livre aident efficacement à démêler les fils d'une 
pensée novatrice, mais parfois obscure en raison de son style poétique et allusif, de ses redondances et qui procède souvent davantage par fulgurations et intuitions que par le recours à l'argumentation rationnelle. Il faut donc savoir gré aux auteurs de cette tentative sans précédent (ou presque) ${ }^{3}$ en langue française pour faire connaître au public francophone l'un des aspects les plus marquants de la pensée de Grundtvig. Il reste évidemment à souhaiter que d'autres études voient bientôt le jour pour révéler les différents aspects de cette œuvre singulière et provocante dont l'apparente dispersion entre des champs hétérogènes trouve au contraire son unité et sa cohérence dans son enracinement théologique original.

\section{NOTES}

1. Ove Korsgaard, professeur émérite à l'université d'Aarhus, est l'un des spécialistes danois de la pensée de Grundtvig. On peut lire de lui, en anglais N.F.S. Grundtvig. As a Political Thinker, Copenhague, Djøf, 2014

2. En Suède par exemple. Voir Georges Ueberschlag, La folkhögskola. Étude de l'évolution historique, idéologique et pédagogique des écoles supérieures d'adultes en Suède, 1868-1945, Atelier de reproduction des thèses, Université Lille III [diffusion Librairie Honoré Champion], 1981, 2 vol.

3. Sur les conceptions éducatives de Grundtvig et la question des hautes études populaires, l'étude fondamentale reste évidemment la thèse d'Erica Simon, Réveil national et culture populaire en Scandinavie, citée en bibliographie. Qui voudrait approfondir sa connaissance de la vie de Grundtvig et des divers aspects de sa pensée pourra se reporter aux publications mentionnées dans les notes et la bibliographie. On pourra y ajouter le seul livre sur Grundtvig jamais publié en France (à ma connaissance) : Hal Koch, Grundtvig, barde et animateur du peuple danois, trad. de M. Metzger, Genève, Labor, s. d. En anglais, on pourra se reporter en particulier aux deux ouvrages suivants : S. A. J. Bradly, N.F.S. Grundtvig, A Life Recalled. An Anthology of Biographical Source-Texts, Aarhus, Aarhus University Press, 2008 ; et A. M. Allchin, N.F.S. Grundtvig. An Introduction to his Life and Work, Aarhus, Aarhus University Press, 1997. Signalons enfin, dans le champ théologique, deux timides apparitions du nom de Grundtvig en France : Eduard Lehmann, « Deux réformateurs du protestantisme danois. Kierkegaard et Grundtvig ", Revue d'histoire et de philosophie religieuses, XI, 1931, p. 499-505 et Vagn Hansen, « La conception ecclésiologique de Grundtvig », Dieu vivant, no 35, 1953, p. 143-146. 